\title{
The thymus in hepatic cirrhosis
}

\author{
MAURICE CORRIDAN
}

From the Department of Pathology, Dewsbury General Hospital, Yorkshire

SYNOPSIS A post-mortem examination, in the case of a 34-year-old man dying suddenly from head injuries sustained in a road accident, showed early cirrhosis of the liver and associated splenomegaly 17 years after an attack of infective hepatitis. The histology of the thymus showed abnormal changes in the medulla, consisting of epithelial hyperplasia and the presence of lymph follicles with germinal centres. It is suggested that an auto-immune mechanism underlay the pathogenesis of the cirrhosis and that the thymic changes were concerned with this.

Recently pathological changes have been described in the thymus in cases of myasthenia gravis and rheumatoid arthritis, diseases of an auto-immune nature (Burnet and Mackay, 1962). Essentially these were the abnormal presence of lymph follicles with germinal centres in various stages of activity and conspicuous epithelial proliferation enlarging the thymic medulla. Burnet (1962) found similar thymic changes in NZB/Bl mice spontaneously developing auto-immune haemolytic anaemia; he believes that they are peculiar to and will be found in many autoimmune diseases. A case of early post-necrotic cirrhosis with similar thymic changes is described here.

\section{CASE REPORT}

A single man, aged 34 , while motor cycling home from his club on the afternoon of 9 December 1962 struck a lamp post. He was quickly removed in an unconscious state to hospital but died 10 minutes after admission. The accident was subsequently shown to have been caused by mechanical locking of the steering due to the remains of part of a broken key.

\section{POST-MORTEM EXAMINATION}

A post-mortem examination was carried out 20 hours after death, the body having been stored in the refrigerator during that period.

Death had been caused by multiple fractures of the skull and haemorrhage and contusion of the brain. The rest of the examination showed the following interesting findings.

The liver was enlarged $3 \mathrm{~cm}$. below the costal margin, weighed $1,950 \mathrm{~g}$., and the surface was irregul- arly and faintly nodular with the largest nodules measuring up to $1 \mathrm{~cm}$. in diameter. The cut surface was pale fawn in appearance and firm. The biliary system was normal. The gross appearance of the liver was that of early cirrhosis. The spleen was thrice normal in size, weighed $530 \mathrm{~g}$., and the sliced surface showed a congested normal pattern. The thymus gland was conspicuous, flesh coloured, and weighed $28 \mathrm{~g}$. The sliced surface of the kidneys and thyroid and the remaining organs showed nothing noteworthy.

\section{PREVIOUS MEDICAL HISTORY}

The previous medical history was obtained from his mother. He enjoyed a normal healthy childhood, left school at the age of 15 , and worked contentedly as a labourer for the rest of his life. At the age of 17 he suffered from infective hepatitis, being as "yellow as a marigold' for eight weeks and spending the last four in hospital. He apparently made a complete recovery and had no further attacks of jaundice. He drank 3-4 pints of beer nightly for many years. He had a normal appetite but only desired a light breakfast of tea and toast.

\section{HISTOLOGY}

Sections of the liver showed chronic active hepatitis and early cirrhosis. The tracts separating the liver nodules of varying size were thin and composed of fibroblastic and fibrous tissue. The largest nodules possessed both portal tracts and centrilobular veins as seen in post-necrotic or post-hepatitis cirrhosis. No fat or alcoholic hyaline was seen in the liver cells. Heavy accumulations of plasma cells and lymphocytes were present in the tracts and were surround- 


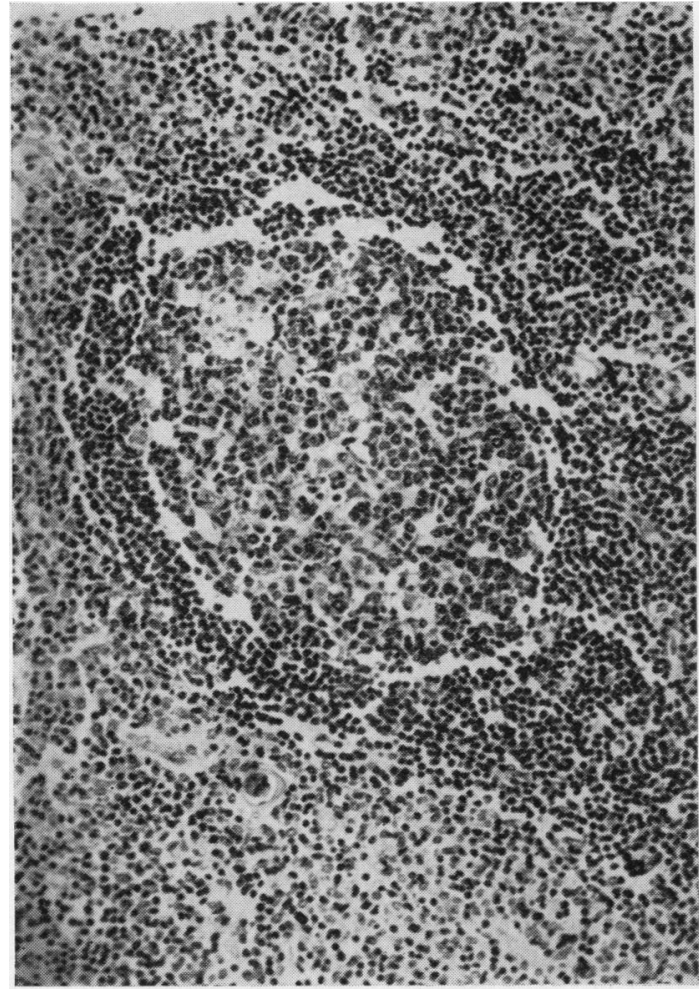

FIG. 1

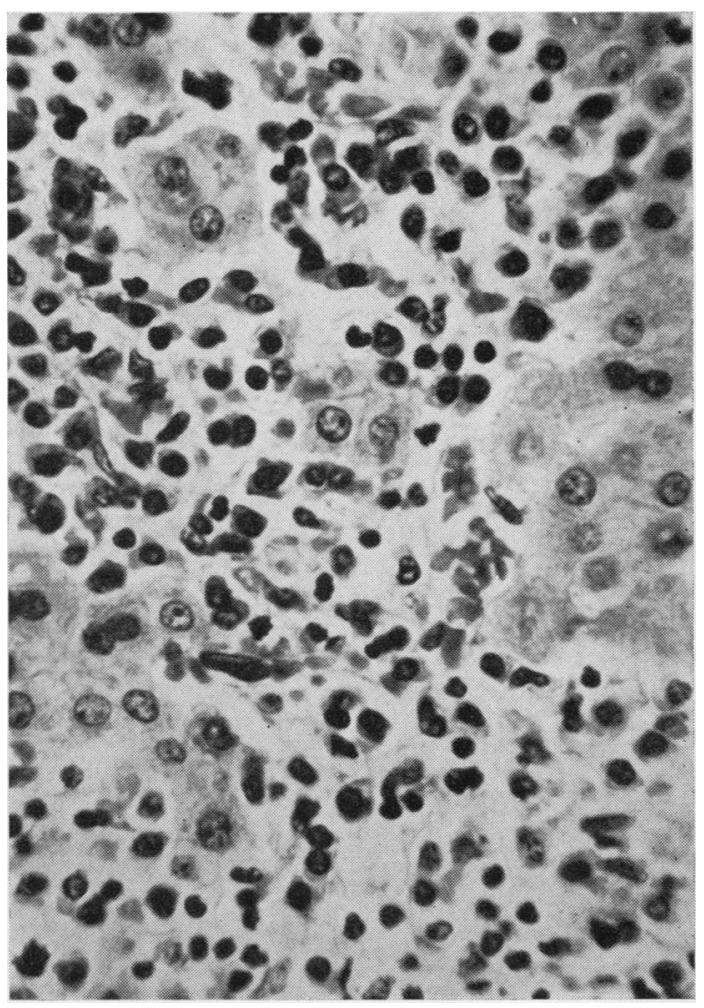

FIG. 2

FIG. 1. Edge of a liver nodule showing plasma cells and lymphocytes confronting and isolating damaged and degenerating liver cells. Haematoxylin and eosin $\times 500$.

FIG. 2. Medulla of thymus showing a lymph follicle with an active germinal centre. Haematoxylin and eosin $\times 170$.

ing and isolating damaged and degenerating liver cells at the edge of the adjacent nodules (Fig. 1). The fibrous tissue formed in the tracts appeared to be an aftermath of this chronic inflammatory reaction.

In the spleen small numbers of Malpighian bodies showed hyperplasia and the formation of germinal centres with mitoses and large pyroninophilic cells. The sinuses of the splenic pulp showed congestion, proliferation of the lining endothelium, and free macrophages with an occasional phagocytosed red cell. Large numbers of mature plasma cells and an occasional eosinophil were present throughout the pulp.

The thymus consisted of hypertrophied lobules separated by varying amounts of adipose tissue representing somewhat less than the expected degree of involution for age. The cortex was prominent and consisted of densely packed roundish masses of darkly staining small lymphocytes. The medulla was increased in width due to the following abnormal features. Many lobules contained lymph follicles with germinal centres surrounded by a narrow zone of small lymphocytes (Fig. 2). Some active centres showed mitoses and pyroninophilic cells were inconspicuous. Hassall's corpuscles in various stages of development were numerous throughout the medulla and were enveloped in wide mantles or surrounded by diffuse areas of proliferating epithelial cells. The rest of the medulla was occupied by proliferating epithelium mingled with lymphoid cells. Plasma or mast cells were not seen.

\section{DISCUSSION}

Auto-antibodies have been found in cases of progressive hepatitis (Mackay and Larkin, 1958; Hijmans, Doniach, Roitt, and Holborow, 1961) and in this case the histological appearance of plasma cells surrounding degenerating liver cells is consistent with a reaction of an immunological nature and suggests the likelihood that an auto-immune mechanism 
underlay the pathogenesis of the chronic hepatitis and cirrhosis. The liver lesion was found 17 years after the patient had apparently made a good recovery from a severe attack of infective hepatitis but it is known that an interval of years of good health may occur before cirrhosis develops (Sherlock, 1958). It is possible that during this clinically silent period the virus of infective hepatitis persists in the body as shown by the case of Creutzfeldt. Schmitt, Richert, Kaiser, and Matthews (1962), where a man was found to be dying of primary carcinoma of liver complicating post-necrotic cirrhosis 17 years after a mild attack of infective hepatitis. For 10 years before his death he had been a blood donor, and eight recipients had definitely developed jaundice after receiving his blood. Since the eight recipients covered a span of 10 years, it is apparent that a persistent or intermittent viraemia lasted during that time. It is thus possible to incriminate the virus and customary to include the alcohol consumed as agents capable of promoting either the continuation of the original liver damage in an apparently slow, silent manner or causing its resumption at some unspecified time during the symptom-free interval. The damaged liver cells would then produce leakage and exposure of normally inaccessible antigen and so initiate its pathogenetic role in stimulating autoantibody formation.

In the spleen the germinal-centre formation in the Malpighian bodies and plasma cell proliferation throughout the pulp closely resemble the splenic changes in immunized animals, and Marshall (1956) states that this combined reaction is produced only by antigenic substances. It is possible to explain the splenic changes on a basis of reactions to antigen derived from the degerating liver cells as suggested by Himsworth (1947), but more difficult on grounds of persistence of the virus in the reticulo-endothelial system as similar splenic reactions are produced by chemical or mechanical liver damage (Marshall, 1956).

The thymic changes are similar to those described in rheumatoid arthritis and myasthenia gravis (Burnet and Mackay, 1962) and an auto-immune component in their pathogenesis is usually accepted. Plasma cells were conspicuously absent in contrast to their heavy accumulation in the liver and spleen. Burnet and Mackay (1962) believe that the process of auto-immune disease is initiated in the thymus, and regard the medullary epithelial hyperplasia and germinal-centre formation as changes reflecting the breakdown in that condition of the immunological homeostatic control normally exercised by the thymus.

I am indebted to Dr. J. J. Fitzpatrick for the medical history; to Dr. A. Fullerton, M.C. for interest and providing a copy of one reference; and to Mr. P. Asquith, F.I.M.L.T., Mr. N. Coward, F.I.M.L.T., and Mr. E. D. Kendall, F.I.M.L.T., for technical assistance.

\section{REFERENCES}

Burnet, Sir M. (1962). Proc. roy. Soc. Med., 55, 619.

$\longrightarrow$, and Mackay, I. R. (1962). Lancet, 2, 1030.

Creutzfeldt, W., Schmitt, H., Richert, J., Kaiser, K., and Matthews, M. (1962). Dtsch. med. Wschr., 87, 1801.

Hijmans, W., Doniach, D., Roitt, I. M., and Holborow, E. J., (1961). Brit. med. J., 2, 909.

Himsworth, H. P. (1947). An Outline of the Liver and its Diseases, p. 14. Blackwell, Oxford.

Mackay, I. R., and Larkin, L. (1958). Aust. Ann. Med., 7, 251.

Marshall, A. H. E. (1956). Cytology and Pathology of the Reticular Tissue, p. 144. Oliver and Boyd, Edinburgh.

Sherlock, Sheila (1958). Diseases of the Liver and Biliary System, p. 263. Blackwell Scientific Publications, Oxford. 